\title{
Über die Zusammensetzung der 1905-er Citronensäfte.
}

\author{
Von
}

\section{A. Beythien, Paul Bohrisch und Hans Hempel.}

Mitteilung aus dem Chemischen Untersuehungsamte der Stadt Dresden.

In der Überzeugung, daß die Analyse des Citronensaftes durch die bahnbrechende Arbeit Farnsteiner's ${ }^{1}$ ) zum ersten Male auf eine wirklich exakte wissenschaftliche Grundlage gestellt worden sei, und daß die Einführung der indirekten Extraktbestimmung für die praktische Nahrungsmittelchemie von hervorragendem Nutzen werden könne, haben wir seit einigen Jahren versucht, durch Untersuchungen selbst hergestellter Säfte aus Früchten bekannter Sorte und Herkunft die Kenntnisse über die durch Art, Witterung und Standort bedingten Schwankungen der Zusammensetzungzu erweitern. In unserer ersten Veröffentlichung ${ }^{2}$ ) berichteten wir über die Analysen von 17 selbstgepreßten Säften aus 8 verschiedenen Citronensorten, deren chemische Eigenschaften im großen und ganzen mit den von Farnsteiner ermittelten Befunden übereinstimmten. Insbesondere kamen die Minimalwerte für den totalen Extraktrest mit 0,984 und 0,936 dem Farnste in er'schen Mindestgehalte 1,00 sehr nahe. Um die durch den wechselnden Grad der Spritung bedingten Unterschiede auszuschalten, empfahlen wir, alle analytischen Daten auf alkoholfreie Substanz umzurechnen, und glaubten, unter dieser Voraussetzung $0,8 \mathrm{~g}$ als äuBerste Grenze für den Extraktrest. natürlicher Citronensäfte vorschlagen zu sollen.

Inzwischen war die zweite Abhandlung $F$ arnsteiner's ${ }^{3}$ ) erschienen, welche die Methode der indirekten Extraktbestimmung in noch konsequenterer Weise durch Berücksichtigung der spezifischen Gewichte aller wichtigeren Bestandteile des Citronensaftes durchführte und im Prinzipe zweifellos eine weitere wesentliche Verbesserung bedeutete. Eine Nachprüfung der Grundlagen des Verfahrens ist bisher nur von L e p èr $\mathrm{e}^{4}$ ) vorgenommen worden, welcher die Anwendbarkeit der Farnste in er'sehen Citronensäuretabelle, sowie der amtlichen Zuckertabelle bestätigte und nur für das spezifische Gewicht der Mineralstofflösungen bei Gegenwart wechselnder Mengen freier Citronensäure andere Zahlen fand. Da auch unsere, allerdings nur vereinzelten, Bestimmungen abweichende Werte für den Mineralstoff-Faktor ergeben haben, so halten wir weitere Untersuchungen über diesen Gegenstand für wünschenswert, haben es aber einstweilen als unbedenklich angesehen, der Berechnung der nachfolgenden Analysen den Faktor 7,15 zugrunde zu legen.

Obwohl in unserer ersten Arbeit deutlich zum Ausdruck gebracht worden ist, dal die dort mitgeteilten Werte für den Extraktrest nach dem ursprünglichen Vorschlage Farusteiner's, d. b. unter ausschließlicher Benutzung seiner CitronensäureTabelle berechnet worden sind, haben doch verschiedene Anfragen von Fachgenossen, sowie die neueren Veröffentlichungen gezeigt, daß die angeführten Minimalzahlen mit den nach dem Additionsverfahren erlangten verwechselt worden sind. Wir haben daher unsere frühere Tabelle nach dem letzteren umgerechnet und dabei folgende Werte erlangt, die nun zu einem direkten Vergleiche mit den Befunden anderer Autoren und unseren eigenen neuen Bestimmungen herangezogen werden können.

1) Diese Zeitschrift $1908,6,1$.

2) Diese Zeitschrift 1905, 9, 449

$\left.{ }^{3}\right)$ Diese Zeitschrift 1904, 8, 593.

4) Zeitsehr. offentl. Chem. 1906, 12, 1 . 
Tabelle I.

Zusammensetzung selbstgeprefiter Citronensafte des Jahres 1904, auf alkoholfreie $S u b s t a n z$ berechnet.

\begin{tabular}{|c|c|c|c|c|c|c|c|c|c|c|}
\hline \multirow[b]{2}{*}{ No, } & \multirow[b]{2}{*}{$\begin{array}{c}\text { Bezeichnung } \\
\text { der Oitronen-Sorte }\end{array}$} & \multirow{2}{*}{$\begin{array}{l}\text { Qesant- } \\
\text { Citro- } \\
\text { nen- } \\
\text { säure, } \\
\text { wasser- } \\
\text { frei } \\
\mathbf{g}\end{array}$} & \multirow{2}{*}{$\begin{array}{l}\text { Gesamt- } \\
\text { zucker, } \\
\text { als } \\
\text { Tnyert- } \\
\text { zucker } \\
\mathrm{g}\end{array}$} & \multirow[b]{2}{*}{$\begin{array}{c}\text { Mine- } \\
\text { ral- } \\
\text { stoffe } \\
\\
\mathrm{g}\end{array}$} & \multirow[b]{2}{*}{$\begin{array}{l}\text { Alkali- } \\
\text { tät } \\
=\text { ecm } \\
\text { N.- } \\
\text { Lauge }\end{array}$} & \multirow[b]{2}{*}{$\begin{array}{l}\text { Stiek- } \\
\text { stoff }\end{array}$} & \multirow[b]{2}{*}{$\begin{array}{l}\text { Phos- } \\
\text { phor- } \\
\text { säure } \\
\text { g }\end{array}$} & \multicolumn{2}{|c|}{ Extrakt } & \multirow[b]{2}{*}{$\begin{array}{c}\text { Totaler } \\
\text { Ex- } \\
\text { trakt- } \\
\text { rest } \\
\\
\mathrm{g}\end{array}$} \\
\hline & & & & & & & & direkt & $\begin{array}{c}\text { nach } \\
\text { der Ad- } \\
\text { ditions- } \\
\text { methode } \\
g\end{array}$ & \\
\hline 1 & Verdelli . . & 6,600 & 1,500 & 0,421 & 5,040 & 0,067 & 0,028 & 8,774 & 9,423 & 0,743 \\
\hline 2 & & 6,660 & 1,490 & 0,416 & 5,200 & 0,067 & 0,026 & 8,782 & 9,459 & 0,720 \\
\hline 3 & Syrakusa I & 6,990 & 1,630 & 0,477 & 5,660 & 0,052 & 0,029 & 9,470 & 10,164 & 0,838 \\
\hline 4 & & 7,710 & 1,490 & 0,508 & 5,800 & 0,056 & 0,027 & 10,007 & 10,652 & 0,751 \\
\hline 5 & Syrakusa III . & 6,430 & 1,840 & 0,413 & 5,140 & 0,045 & 0,030 & 8,804 & 9,662 & 0,808 \\
\hline 6 & & 6,570 & 0,060 & 0,413 & 4,990 & 0,038 & 0,029 & 7,635 & 7,839 & 0,629 \\
\hline 7 & Syrakusa II & 6,960 & 1,340 & 0,406 & 5,350 & 0,048 & $0,020^{\circ}$ & 9,415 & 9,726 & 0,842 \\
\hline 8 & . & 6,820 & 1,680 & 0,417 & 5,850 & 0,047 & 0,021 & 9,199 & 9,767 & 0,655 \\
\hline 9 & $\pi$ & 7,070 & 0,050 & 0,402 & 5,890 & 0,046 & 0,021 & 8,167 & 8,316 & 0,614 \\
\hline 10 & Catania. & 7,240 & 1,290 & 0,505 & 5,820 & 0,054 & 0,020 & 9,164 & 9,971 & 0,742 \\
\hline 11 & & 7,550 & 0,540 & 0,535 & 6,580 & 0,046 & 0,021 & 9,139 & 9,667 & 0,823 \\
\hline 12 & Messina & 6,850 & 1,470 & 0,491 & 6,280 & 0,049 & 0,029 & 9,149 & 9,719 & 0,699 \\
\hline 13 & & 7,000 & 0,040 & 0,457 & 5,690 & 0,041 & 0,028 & 8,128 & 8,361 & 0,674 \\
\hline 14 & Malaga I. & 6,950 & 1,820 & 0,563 & 7,230 & 0,059 & 0,020 & 10,014 & 10,740 & 0,966 \\
\hline 15 & $n$. & 6,680 & 0,011 & 0,566 & 7,380 & 0,049 & 0,019 & 8,228 & 8,294 & 0,841 \\
\hline 16 & Malaga II . & 6,600 & 2,550 & 0,576 & 7,340 & 0,073 & 0,031 & 10,164 & 10,886 & 0,915 \\
\hline 17 & $\pi$. & 6,680 & 0,080 & 0,598 & 7,470 & 0,062 & 0,028 & 8,452 & 8,607 & 1,000 \\
\hline & Mittel & 6,900 & - & 0,480 & 6,012 & 0,053 & 0,025 & 8,982 & $9,48 \mathbf{5}$ & 0,779 \\
\hline & Höchst & 7,710 & 2,550 & 0,598 & 7,470 & 0,073 & 0,081 & 10,164 & 10,886 & 1,000 \\
\hline & Niedrigst & 6,430 & 0,011 & 0,402 & 4,990 & 0,038 & 0,019 & 7,635 & 7,839 & 0,614 \\
\hline
\end{tabular}

Hieraus geht hervor, daß die von uns untersuchten Citronensäfte des Jahres 1904 nach der Additionsmethode einen totalen Extraktrest von 0,614 bis 1,00, im Mittel von 0,779 aufwiesen, und daß die Differenz gegen die nach dem ursprünglichen Verfahren berechneten Werte 0,254 bis 0,543 beträgt. Im Hinblick auf diese, vom Mineralstoffgehalt beeinflußten, ziemlich erheblichen Schwankungen ist es nicht angängig, wie wir damals meinten, die älteren Werte einfach um 0,4 zu erniedrigen. Vielmehr erscheint die prinzipielle Anwendung der Additionsmethode zur Erlangung brauchbarer Ergebnisse unerläßliche Voraussetzung.

Nach den bei anderen Fruchtsäften, besonders bei den Himbeersäften des letzten Jahres, gemachten Erfahrungen konnte es keinem Zweifel unterliegen,-daß das vorhandene analytische Material zu einer endgültigen Entscheidung über die Zusammensetzung des Citronensaftes nicht ausreichte, und wir begrüßten es daher freudig, als Lührig auf einer zu Anfang März d. J. in Dresden abgehaltenen Versammlung von Fachgenossen einige vorläufige Mitteilungen über die Untersuchung selbstgepreßter Citronensäfte zum Vortrag brachte. Auf die soeben im Druck erschienene Abhandlung ${ }^{1}$ ) kommen wir noch zurück, möchten aber zuvor über die Ergebnisse unserer

1) Diese Zeitschrift 1906, 11, 441. 
eigenen Untersuchungen, die wir, angeregt durch die von Lührig erlangten abweichenden Ergebnisse, alsbald in Angriff nahmen, berichten.

Um über die Herkunft und Sorte der verarbeiteten Früchte völlig im klaren zu sein, setzten wir uns wieder mit derselben angesehenen Firma in Verbindung, welche uns in freundlichster Weise das Ausgangsmaterial unserer früheren Arbeit verschafft hatte; wir gelangten durch sie in den Besitz folgender Citronensorten: 1 Sorte Neapeler Insel-Citronen, 2 Sorten Syrakusa-, 2 Sorten Messina-, 2 Sorten Sorrento-, 2 Sorten Neapeler Garten-Gitronen.

Die Früchte hatten in bezug auf Gestalt, Gewicht und Saftgehalt folgende Eigenschaften:

1. Neapeler Insel-Citronen, von den umliegenden kleinen Inseln in der Nähe von Neapel. Fast kugeIrunde Früchte mit dunkelgelber glatter Schale. Durchschnittsgewicht $175 \mathrm{~g}$; ohne Fruchtschale und Pulpa $106 \mathrm{~g}$. 20 Früchte gaben $1010 \mathrm{ecm}$ Saft.

2. Syrakusa I. Kleine längliche Früchte von hellgelber Farbe. Durchschnittsgewicht einer Frucht $93 \mathrm{~g}$; nach Entfernung der Fruchtschale und Pulpa $55 \mathrm{~g}$. 20 Früchte gaben $393 \mathrm{ccm}$ Saft.

3. Syrakusa II. Mittelgrofe gelbe Früchte Durchschnittsgewicht einer Frucht $116 \mathrm{~g}$; ohne Fruchtschale und Pulpa $70 \mathrm{~g}$. 20 Erüchte gaben $923 \mathrm{ccm}$ Saft.

4. M es sina I. Kleine hochgelbe Früchte. Durchschnittsgewicht $107 \mathrm{~g}$, ohne Fruchtschale und Pulpa 68,5 g. 20 Früchte gaben $983 \mathrm{ccm}$ Saft.

5. Messina II. Rundliche hellgelbe Früchte. Durchschnitsgewicht einer Frucht $117 \mathrm{~g}$; ohne Fruchtschale und Palpa $76 \mathrm{~g}$. 20 Früchte gaben $1220 \mathrm{ccm}$ Saft.

6. Sorrento I. Sehr große, länglich geformte Früchte mit großen Höckern, hellgelber mit tiefen Grübchen versehener Schale und starken. breiten Blättern. Citronen von wundervollem Aroma und Anssehen. Durchschnittsgewicht einer Frucht $312 \mathrm{~g}$ ohne Fruchtschale und Pulpa $140 \mathrm{~g}$. 20 Früchte gaben $1800 \mathrm{ccm}$ Saft.

7. Sorento II. Von ähnlich schönem Aussehen wie vorige Probe, aber mit kleineren Blättern und oft birnenförmiger Gestalt. Durchschnittsgewicht einer Frucht $265 \mathrm{~g}$; ohne Fruchtschale und Pulpa $122 \mathrm{~g}$. 20 Früchte gaben $1870 \mathrm{ccm}$ Saft.

8. und 9. Neapeler Garten-Citronen. Ziemlich grobe, teils glatte, teils höckerige Früchte mit hochgelber Schale und mittelgroßen, länglichen Blättern. Durchschnittsgewicht einer Frucht $184 \mathrm{~g}$; ohne Fruchtschale and Pulpa $100 \mathrm{~g} .20$ Früchte gaben $1247 \mathrm{ccm}$ Saft.

Die von Schale und Pulpa sorgfältig befreiten Früchte wurden kräftig ausgepreßt, und die abfließenden Säfte in offenen tarierten Flaschen 8 Tage lang bei einer auch während der Nacht gleichmäBig anf $30^{\circ} \mathrm{C}$ erhaltenen Temperatur aufbewahrt. Nach Beendigung der lebhaft einsetzenden und unter starkem Schäumen verlaufenden Gärung wurden die Flüssigkeiten mit destilliertem Wasser zum ursprünglichen Gewichte ergänzt, durch Gaze koliert und mit 10 Vol..\% Alkohol vermischt. Die einige Tage später nach dem Absitzen der Pektinstoffe ohne Anwendung von Klärungsmitteln in der Kälte filtrierten Säfte erschienen völlig klar, besaßen prachtvolles Aroma und erhielten sich bis zum heutigen Tage völlig unverändert.

Die Analyse erfolgte wie bei unserer früheren Arbeit nach dem von Farnsteiner vorgeschlagenen Gange. Nur erschien es auf Grund einiger neuer, weiter unten mitgeteilter Erfahrungen ratsam, in allen Fällen auch die flüchtigen Säuren quantitativ zu bestimmen, obwohl deren Menge in den selbstgepreBten Säften naturgemäß nur gering sein konnte. Außerdem wurde der Gehalt an sog. Glycerin ermittelt, und vor allem die in Form von Estern vorhandene Citronensäure durch Ver- 
seifung mit Natronlauge bestimmt. Nach unseren letzten Beobachtungen geht ein kleiner Teil der Citronensäure bei energischem Verlaufe der Gärung und nachfolgendem Alkoholzusatz schon innerhalb kurzer Zeit eine Verbindung mit dem Alkohol ein und entzieht sich der direkten Titration, und Werte von 0,15 und $0,16 \mathrm{~g}$ gebundener Citronensäure. die wir in frischgepreßten Säften auffanden, dürfen bei dieser peniblen Methode nicht außer acht gelassen werden. Wie schnell die Veresterung der Citronensäure vor sich geht, mögen nachfolgende Zahlen veranschaulichen:

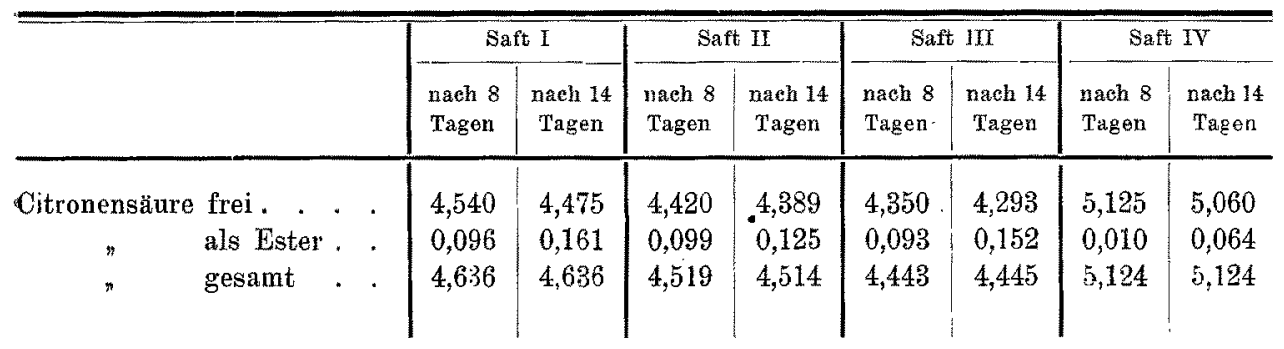

Tabelle

Selbstgeprefte Citronensäfte

\begin{tabular}{|c|c|c|c|c|c|c|c|}
\hline \multirow{3}{*}{ No. } & \multirow{3}{*}{$\begin{array}{l}\text { Bezeichnung } \\
\text { der Sorten }\end{array}$} & \multirow{2}{*}{\multicolumn{2}{|c|}{$\begin{array}{c}\text { Spezifisehes Gewicht } \\
\text { des entgeisteton } \\
\text { Saftes }\end{array}$}} & \multirow{3}{*}{$\begin{array}{c}\text { Freie } \\
\text { Gesant- } \\
\text { säure als } \\
\text { Citronen- } \\
\text { säure } \\
\mathrm{g}\end{array}$} & \multicolumn{2}{|c|}{ Fǘchtige Säuren } & \multirow{3}{*}{$\begin{array}{c}\text { Freie } \\
\text { Citronen- } \\
\text { säure } \\
\\
\mathrm{g}\end{array}$} \\
\hline & & & & & \multirow{2}{*}{$\begin{array}{c}\text { a } \\
\text { als } \\
\text { Essig- } \\
\text { süure } \\
\text { g }\end{array}$} & \multirow{2}{*}{$\begin{array}{c}\mathrm{b} \\
\text { als } \\
\text { Citronen- } \\
\text { süure } \\
\mathrm{g}\end{array}$} & \\
\hline & & $\begin{array}{c}a \\
\text { direkt }\end{array}$ & $\begin{array}{c}\text { b } \\
\text { korrigiert }\end{array}$ & & & & \\
\hline 1 & Neapeler Insel-Citronen. & 1,02585 & 1,02606 & 4,475 & 0,045 & 0,046 & 4,429 \\
\hline 2 & Syrakusa I . . . . & 1,03343 & 1,03853 & 5,730 & 0,023 & 0,023 & 5,707 \\
\hline 3 & $\pi$ II . & 1,02907 & 1,02921 & 5,250 & 0,010 & 0,010 & 5,240 \\
\hline 4 & Messina I . & 1,03176 & 1,03191 & 5,630 & 0,030 & 0,031 & 5,599 \\
\hline 5 & $"$ II . & 1,03143 & 1,08158 & 5,820 & 0,030 & 0,081 & 5,789 \\
\hline 6 & Sorrento I & 1,02404 & 1,02421 & 4,389 & 0,045 & 0,046 & 4,343 \\
\hline 7 & , II . . . . . . & 1,02360 & 1,02380 & 4,298 & 0,028 & 0,023 & 4,270 \\
\hline 8 & Neapeler Garten-Citronen I. & 1,02795 & 1,02807 & 5,060 & 0,023 & 0,023 & $5,0.37$ \\
\hline \multirow[t]{4}{*}{9} & $\because \quad, \quad$ II & 1,02512 & 1,02523 & 4,420 & 0,010 & 0,010 & 4,420 \\
\hline & Mittel & 1,02803 & 1,02817 & 5,007 & 0,027 & 0,027 & 4,983 \\
\hline & Höchst & 1,03343 & 1,03353 & 5,820 & 0,045 & 0,046 & 5,789 \\
\hline & Niedrigst & 1,02360 & 1,02380 & 4,293 & 0,010 & 0,010 & 4,270 \\
\hline
\end{tabular}

Bei einer Betrachtung der in Tabelle-III zusammengestellten Analysenbefunde springen zunächst die auberordentlichen Unterschiede in der Zusammensetzung der hier verzeichneten 9 Säfte in die Augen, Unterschiede, die besonders auffallend bei Probe 2 einerseits und Proben 6 und 7 andererseits erscheinen. Während der totale Extraktrest bei No. 2 fast doppelt so hoch ist wie der Mittelwert, erreicht er bei No. 6 und 7 nur die Hälfte des Durchschnittes, und in ähnlicher, wenngleich weniger ausgeprägter Weise deuten die übrigen Bestandteile darauf hin, đaß No. 2 ein stark konzentrierter, No. 7 aber ein sehr dünner Saft ist. Eine Erklärung für dieses merkwürdige Verhalten gibt uns die Berücksichtigung des Gewichtes und Saftgehaltes der einzelnen Citronensorten, indem die niedrigen Extraktreste der Sorrento-Citronen mit 
Zur Vermeidung des durch diese Veränderlichkeit bedingten Fehlers haben wir freie und veresterte Citronensäure sowie spezifisches Gewicht des eingedampften Saftes stets gleichzeitig bestimmt. Die Berechnung des an je $1 \mathrm{~g}$ veresterte Citronensäure gebundenen Alkohols erfolgte unter Benutzung der Zahl 0,719, statt des von Farns teiner anscheinend irrtümlich mitgeteilten Faktors 0,742, und durch Subtraktion des entsprechenden spezifischen Gewichtes wurden dann die in Tabelle II als "Spezifisches Gewicht, korrigiert" bezeichneten Werte erhalten.

Schließlich haben wir im Hinblick auf die bereits erwähnte Arbeit von Lie père auch den Extrakt direkt nach der Weinvorschrift auf die Weise ermittelt, daß nicht mehr als $1,5 \mathrm{~g}$ zur Wägung gelangte.

Die chemische Zusammensetzung der Säfte ergibt sich aus den Tabellen II und III (S. 656), von denen letztere die auf alkoholfreie Substanz umgerechneten Werte enthält.

II.

aus Citronen des Jahres 1905.

\begin{tabular}{|c|c|c|c|c|c|c|c|c|c|c|}
\hline \multirow[b]{2}{*}{$\begin{array}{c}\text { Citronen- } \\
\text { säure ats } \\
\text { Ester }\end{array}$} & \multirow[b]{2}{*}{$\begin{array}{l}\text { Gesamt- } \\
\text { Citronen- } \\
\text { säure } \\
\mathrm{g}\end{array}$} & \multirow[b]{2}{*}{$\begin{array}{c}\text { Gesamt- } \\
\text { zucker als } \\
\text { Invert- } \\
\text { zucker } \\
\mathrm{g}\end{array}$} & \multirow[b]{2}{*}{$\begin{array}{c}\begin{array}{c}\text { Mineral- } \\
\text { stoffe }\end{array} \\
\qquad \mathrm{g}\end{array}$} & \multirow[b]{2}{*}{$\begin{array}{l}\text { Alkalität } \\
\text { derselben } \\
=\text { com } \\
\text { N.-Lauge }\end{array}$} & \multirow[b]{2}{*}{$\begin{array}{l}\text { Stickstoff } \\
\mathrm{g}\end{array}$} & \multirow[b]{2}{*}{$\begin{array}{c}\text { Glycerin } \\
\mathrm{g}\end{array}$} & \multirow[b]{2}{*}{$\begin{array}{c}\text { Alkohol } \\
g\end{array}$} & \multicolumn{2}{|c|}{ Extraikt } & \multirow[b]{2}{*}{$\begin{array}{c}\text { Totaler } \\
\text { Ex- } \\
\text { trakt- } \\
\text { rest } \\
8 \\
8\end{array}$} \\
\hline & & & & & & & & $\begin{array}{c}\underset{\mathrm{a}}{\mathrm{dir} e \mathrm{k} t} \\
\mathrm{~g}\end{array}$ & $\begin{array}{c}b \\
\text { naeb } \\
\text { der Ad- } \\
\text { ditions- } \\
\text { methode } \\
g \\
g\end{array}$ & \\
\hline 0,161 & 4,590 & 0,173 & & & 0,032 & & 7,072 & 6,082 & 5,874 & 0,561 \\
\hline 0.06 & & & & & & & & 7,5 & & 18 \\
\hline 0,102 & & & & & 0,083 & & 65 & 6,73 & 22 & 0,538 \\
\hline 0,115 & 5,714 & 0,218 & 513 & & 0,044 & 0,098 & 1 & 7,360 & 7,188 & 0,581 \\
\hline 0,080 & & 0,080 & 476 & & 0,031 & 5 & 804 & 7,360 & 7,014 & 0,501 \\
\hline 0,125 & 4,468 & 0,081 & 0.375 & 5,350 & 0,026 & 0,065 & 6,984 & 5,482 & 5,409 & 0,356 \\
\hline 0,152 & 4,422 & & 0,373 & & 0,025 & & 6,960 & 5,468 & 5,294 & 0,284 \\
\hline 0,064 & 5,101 & & 0,408 & & 0,045 & 0,103 & 6,990 & 6,398 & 6,313 & 0,525 \\
\hline 0,083 & 4,503 & 0,030 & 0,384 & 4,968 & 0,050 & - & 7,460 & 5,602 & 5,630 & 0,548 \\
\hline 0,105 & & & & & & & 7,140 & 6,441 & 6,335 & 0,546 \\
\hline 0,161 & 5,869 & & & & 0,05 & & 7,702 & 7,538 & 7,625 & 1,018 \\
\hline 0,061 & 4,422 & 0,030 & 0,362 & 4,872 & 0,025 & $\left.0,05^{\circ}\right)$ & 6,804 & 5,468 & 5,294 & 0,284 \\
\hline
\end{tabular}

dem Durchschnittsgewicht einer Frucht von $312 \mathrm{~g}$ und der kolossalen Saftausbeute von 1800 bis $1870 \mathrm{ccm}$ aus 20 Citronen zusammenfällt, während dem konzentrierten Safte der Probe Syrakusa I ein mittleres Gewicht einer Frucht von $93 \mathrm{~g}$ und eine Saftausbeute von nur $393 \mathrm{ccm}$ entspricht.

Wichtiger erscheint nun der Vergleich der Tabellen I und III, welcher zunächst lehrt, daß die im Jahre 190 o untersuchten Citronensäfte im großen und ganzen tatsächlich etwas dünner zu sein scheinen als diejenigen des Vorjahres. Denn wenn auch Mineralstoffe und Alkalität ziemlich unverändert sind, bleiben doch Citronensäure, Stickstoff und Extraktrest deutlich zurück, und insbesondere beträgt der Mittelwert für den totalen Extraktrest im Jahre 1905 nur 0,597 g gegen 0,779 g im Jahre 1904.

[Fortsetzung S. 657.] 
Tabelle III.

Zusammensetzung selbstgeprefter Citronensafte des Jahres 1905 , anf alkoholfreie Substanz berechnet.

\begin{tabular}{|c|c|c|c|c|c|c|c|c|c|c|}
\hline \multirow[b]{2}{*}{ 5o. } & \multirow[b]{2}{*}{$\begin{array}{c}\text { Bezeiehnung } \\
\text { der Citronen-Sorte }\end{array}$} & \multirow{2}{*}{$\begin{array}{c}\text { Gesamt- } \\
\text { Citro- } \\
\text { nen- } \\
\text { säure, } \\
\text { wasser- } \\
\text { frei } \\
\mathrm{g}\end{array}$} & \multirow[b]{2}{*}{$\begin{array}{l}\text { Gesamt- } \\
\text { zueker } \\
\text { als } \\
\text { Invert- } \\
\text { zucker } \\
\text { g }\end{array}$} & \multirow[b]{2}{*}{$\begin{array}{c}\text { Mine- } \\
\text { ral- } \\
\text { stoffe }\end{array}$} & \multirow[b]{2}{*}{$\begin{array}{l}\text { Alkali- } \\
\text { that } \\
=\text { eem } \\
\text { N.- } \\
\text { Lauge }\end{array}$} & \multirow[b]{2}{*}{$\begin{array}{l}\text { Stiek- } \\
\text { stoff }\end{array}$} & \multirow[b]{2}{*}{$\begin{array}{l}\text { Gly- } \\
\text { cerin }\end{array}$} & \multicolumn{2}{|c|}{ Extrakt } & \multirow[b]{2}{*}{$\begin{array}{c}\text { Totaler } \\
\text { Ex- } \\
\text { trakt- } \\
\text { rest } \\
\\
\mathrm{g}\end{array}$} \\
\hline & & & & & & & & direkt & $\begin{array}{c}\text { nach } \\
\text { der Ad- } \\
\text { ditions- } \\
\text { me- } \\
\text { thode } \\
\mathrm{g}\end{array}$ & \\
\hline 1 & apeler Insel-Citronen . & & 0,190 & 0,397 & 6,203 & 0,035 & 0,129 & 6,619 & 6,448 & 0,616 \\
\hline 2 & usa 1 & 6,388 & 0,222 & 0,518 & 5,682 & 0,051 & 0,1 & 8,348 & 8,445 & 1,128 \\
\hline 3 & II & 5,872 & 0,111 & 0,475 & 5,574 & 0,036 & 0,151 & 7,404 & 7,235 & 0,556 \\
\hline 4 & Messina I & 6,278 & 0,240 & 0,564 & 5,354 & 0,048 & 0,108 & 8,088 & 7,899 & 0,639 \\
\hline 5 &,$\quad$ II & 6,420 & 0,110 & 0,521 & 5,839 & 0,034 & 0,104 & 8,050 & 7,770 & 0,548 \\
\hline 6 & Sorrento I & 4,899 & 0,034 & 0,412 & 5,866 & 0,029 & 0,071 & 6,011 & 5,931 & 0,391 \\
\hline 7 & $\pi$ II . & 4,847 & 0,033 & 0,409 & 6,084 & 0,027 & 0,057 & 5,994 & 5,803 & 0,311 \\
\hline 8 & Neapeler f I & 7,010 & 0,097 & 0,447 & 6,295 & 0,049 & 0,113 & 7,011 & 6,923 & 0,576 \\
\hline 9 & Garten-Citronen III. & 4,700 & 0,033 & 0,424 & 5,484 & 0,055 & - & 6,183 & 6,214 & 0,605 \\
\hline & Mittel & 5,717 & 0,1 & 0,463 & 5,820 & 0,040 & 0,105 & 7,078 & 6,963 & 0,597 \\
\hline & Höcl & 7,019 & 0,2 & 0,564 & 6,259 & 0,055 & 0,151 & 8,348 & 8,445 & 1,12 \\
\hline & Niedrigst & 4,700 & 0,033 & 0,397 & 5,354 & 0,027 & 0,057 & 5,994 & 5,808 & 0,311 \\
\hline
\end{tabular}

Tabelle

Zusammensetzung angeblich, garantiert naturreiner Citronen-

\begin{tabular}{|c|c|c|c|c|c|c|}
\hline \multirow{3}{*}{ No. } & \multirow{3}{*}{ Bézeichnung } & \multirow{2}{*}{\multicolumn{2}{|c|}{$\begin{array}{l}\text { Spezif. Gewicht } \\
\text { des entgeisteten } \\
\text { Saftes }\end{array}$}} & \multirow{3}{*}{\begin{tabular}{|c} 
Freie \\
Gesamt- \\
săaure \\
als \\
Oitro- \\
nen- \\
säure \\
$\mathrm{g}$
\end{tabular}} & \multicolumn{2}{|c|}{$\begin{array}{l}\text { Flïchtige } \\
\text { Säuren }\end{array}$} \\
\hline & & & & & \multirow{2}{*}{$\begin{array}{c}\text { a } \\
\text { als } \\
\text { Essig- } \\
\text { săure } \\
\mathrm{g}\end{array}$} & \multirow{2}{*}{$\begin{array}{c}\mathbf{b} \\
\text { als } \\
\text { Citro- } \\
\text { nen- } \\
\text { säure } \\
\text { g }\end{array}$} \\
\hline & & direkt & korrigiert & & & \\
\hline \multirow[t]{2}{*}{1} & $\begin{array}{l}\text { Versandfertiger Citronensaft. Verschnitt ans } 85 \% \\
\text { Citronen Dezember } 1905 \text { and } 15 \% \text { Citronen }\end{array}$ & & & & & \\
\hline & Juni 1905 (Messina) . . . . . . . , . . & 1,08595 & 1,03595 & 5,957 & 0,334 & 0,356 \\
\hline 2 & Früchte-Ernte November 1905 . & 1,04210 & 1,04228 & 7,624 & 0,277 & 0,296 \\
\hline 3 & Früchte-Ernte Dezember 1905 . & 1,03585 & 1,08555 & 6,666 & 0,072 & 0,077 \\
\hline 4 & Früchte-Ernte Januar 1906 . . . . . . . . & 1,08041 & 1,03113 & 5,289 & 0,017 & 0,018 \\
\hline 5 & $\begin{array}{l}\text { Handelsfertiger Citronensaft, aus 1905-er Früch- } \\
\text { ten, salicyliert. . . . . . . . . . . . }\end{array}$ & 1,03631 & 1,03671 & 5,871 & 0,027 & 0,029 \\
\hline 6 & Desgleichen, gespritet. . . . . . . . . & 1,02679 & 1,02696 & 4,386 & 0,341 & 0,963 \\
\hline 7 & Desgleichen, salicyliert & 1,03623 & 1,08668 & 6,240 & 0,083 & 0,085 \\
\hline 8 & Früchte-Ernte Juni 1905 . . & 1,03245 & 1,03268 & 6,270 & 0,205 & 0,219 \\
\hline \multirow[t]{4}{*}{9} & Früchte-Ernte Dezernber 1905 . . . . & 1,08349 & 1,03349 & 5,326 & 0 & 0 \\
\hline & Mittel & 1,08434 & 1,08459 & 5,959 & 0,145 & 0,155 \\
\hline & Höchst & 1,04210 & 1,04228 & 7,624 & 0,341 & 0,363 \\
\hline & Niedrigst & 1,02679 & 1,02696 & 4,886 & 0 & 0 \\
\hline
\end{tabular}

Essigstichige Citronen-

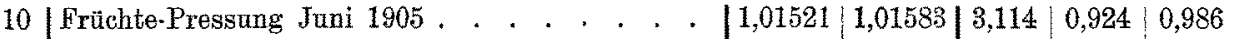

11 Handelsfertiger Citronensaft aus 1905-er Früch-

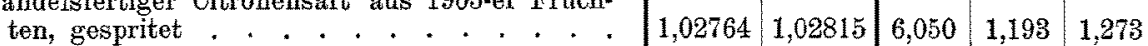
\begin{tabular}{l|l|l|l|l|l|}
\hline 1,02318 & 1,02376 & 4,122 & 1,281 & 1,366
\end{tabular} 
[Fortsetzung von S. 655.]

Immerhin sind die Unterschiede, mit Ausnahme der Proben No. 6 und 7, verhältnismäßig unbedeutend, so daß der Minimalwert von 1904 mit $0,614 \mathrm{~g}$ nur wenig, nämlich um $0,066 \mathrm{~g}$ unterschritten wird.

Würden also die beiden Proben Sorrento-Citronen nicht sein, so brauchten die bisherigen Grundlagen der Beurteilung von Handels-Citronensäften keine allzugroße Abänderung zu erleiden. Es fragt sich, ob diese Säfte ausgeschaltet werden dürfen.

Verschiedene Umstände scheinen mir für die Bejahung dieser Frage zu sprechen. Schon der hohe Preis der wundervollen Sorrento-Citronen, welche in Dresden mit 20 bis $25 \mathrm{Pfg}$ pro Stück bezahlt werden, macht sie wenig geeignet für die Herstellung eines so billigen Massenverbrauchsartikels wie des Citronensaftes, und in der Tat ist uns von fachmännischer Seite versickert worden, daß sie hierfür gar nicht in Frage kommen. Vor allem aber wurden wir in unserer Ansicht bestärkt durch die Untersuchung einer Anzahl von gebrauchsfertigen Handelssäften aus Citronen des letzten Jahres, welche uns von einigen Fruchtpressereien mit größter Bereitwilligkeit zur Verfügung gestellt wurden. Wenn wir für die Reinheit dieser Säfte auch keine unbedingte Garantie übernehmen können, so besteht doch andererseits kein Grund, in die Bürgschaft der uns bekannten Firmen Zweifel zu setzen, Es erscheint daher nicht unberechtigt, die erlangten Untersuchungsbefunde. natürlich unter allem Vorbehalt, in Tabelle IV wiederzugeben.

IV.

säfte des Handels, aus Citronen des Jahres 1905 geprefit.

\begin{tabular}{|c|c|c|c|c|c|c|c|c|c|c|c|c|}
\hline \multirow[b]{2}{*}{$\begin{array}{c}\text { Freie } \\
\text { Citro- } \\
\text { nen- } \\
\text { säure } \\
\\
\mathrm{g}\end{array}$} & \multirow[b]{2}{*}{$\begin{array}{c}\text { Citro" } \\
\text { nen- } \\
\text { säure } \\
\text { als } \\
\text { Ester } \\
\mathrm{g}\end{array}$} & \multirow[b]{2}{*}{$\begin{array}{c}\text { Cesamt } \\
\text { Citro- } \\
\text { nen- } \\
\text { säure } \\
\mathbf{g}\end{array}$} & \multirow[b]{2}{*}{$\begin{array}{c}\text { Gesamt- } \\
\text { zucker } \\
\text { als } \\
\text { Invert- } \\
\text { zucker } \\
\mathbf{g}\end{array}$} & \multirow[b]{2}{*}{$\begin{array}{c}\begin{array}{c}\text { Mine- } \\
\text { ral- } \\
\text { stoffe }\end{array} \\
\mathrm{g}\end{array}$} & \multirow[b]{2}{*}{$\begin{array}{l}\text { Alkali- } \\
\text { tät der- } \\
\text { selben } \\
=\text { com } \\
\text { No- } \\
\text { Lauge }\end{array}$} & \multirow[b]{2}{*}{$\begin{array}{c}\text { Stick- } \\
\text { stoff }\end{array}$} & \multirow[b]{2}{*}{$\begin{array}{l}\text { Gly- } \\
\text { cerin } \\
\mathrm{g}\end{array}$} & \multirow[b]{2}{*}{$\begin{array}{l}\text { Alkohol } \\
\text { Vol.-0\% }\end{array}$} & \multicolumn{2}{|c|}{ Extrakt } & \multirow[b]{2}{*}{$\begin{array}{l}\text { Totaler } \\
\text { Ex- } \\
\text { trakt- } \\
\text { rest } \\
\text { g }\end{array}$} & \multirow[b]{2}{*}{ 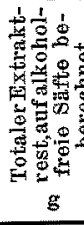 } \\
\hline & & & & & & & & & $\begin{array}{c}a \\
\text { direkt }\end{array}$ & $\begin{array}{c}\mathrm{b} \\
\text { nachder } \\
\text { Addi- } \\
\text { tions- } \\
\text { me- } \\
\text { thode } \\
\mathrm{g}\end{array}$ & & \\
\hline 5,601 & 0 & 5,601 & 1,354 & 0,435 & 5,607 & 0,025 & $0,16 \Omega$ & 0,27 & 8,040 & 8,282 & 0,705 & 0,7 \\
\hline 7,328 & 0,137 & 7,465 & 1,665 & 0,305 & 3.876 & 0,022 & 0,109 & 0 & 9,494 & 9,949 & & \\
\hline 6,589 & 0,158 & 6,742 & 0,160 & 0,432 & 5,755 & 0,043 & 0,104 & 0,74 & 8,313 & 8,100 & 0,574 & \\
\hline 5,271 & 0,529 & 5,800 & 0,123 & 0,394 & 5,535 & 0,044 & 0,103 & 1,11 & 7,004 & 7,077 & 0,576 & \\
\hline 5,842 & 0,285 & 6,127 & 0,488 & 0,424 & 5,6 & 0,048 & & 0,47 & 8,411 & 8,473 & & \\
\hline 4,023 & 0,188 & 4,212 & 0,821 & 0,345 & 4,4 & 0,085 & & 14,61 & 5,987 & 6,200 & 0,6 & \\
\hline 6,205 & 0,290 & 6,495 & 0,642 & 0,439 & 5,5 & 0,046 & 0,113 & 0,48 & 8,497 & 8,390 & 0,6 & \\
\hline 6,051 & 0,177 & 6,228 & 0,128 & 0,378 & & 0,025 & - & 2,32 & 7,402 & 7,474 & 0,589 & \\
\hline 5,326 & 0 & 5,326 & 1,220 & 0,467 & 4,740 & 0,039 & 0,108 & 5,49 & 8,006 & 7,673 & 0,502 & \\
\hline 5,804 & & & & & & & & - & & 7,957 & & \\
\hline 7,328 & 0,529 & 7,465 & 1,665 & 0,467 & 5,755 & 0,048 & 62 & - & 9,494 & 9,949 & 1,246 & 1,2 \\
\hline 4,023 & 0 & 4,212 & 0,123 & 0,305 & 3,876 & 0,022 & 0,047 & - & 5,987 & 6,200 & 0,384 & 0,3 \\
\hline
\end{tabular}

säfte des Handels.

\begin{tabular}{|c|c|c|c|c|c|c|c|c|c|c|c|c}
2,128 & 0,4507 & 2,585 & 0,891 & 0,363 & 4,356 & 0,033 & 0,120 & 0 & 3,310 & - & - & - \\
4,777 & 0,370 & 5,147 & 0,915 & 0,868 & 4,440 & 0,037 & 0,062 & 10,34 & 6,245 & - & - & - \\
\hline 2,756 & 0,427 & 3,183 & 1,365 & 0,395 & 4,820 & 0,029 & - & 0 & 5,166 & - & - & -
\end{tabular}


Von den hier aufgeführten 12 Citronensäften zeigen die ersten 9, mit Ausnahme von No. 2, ganz normale Zusammensetzung. Thre Extraktreste bewegen sich zwischen 1,255 und $0,559 \mathrm{~g}$, so daß der niedrigste Wert hinter demjenigen des Vorjahres nur um 0,075 zurïekbleibt.

Gänzlich abweichend sind nur die Proben No.10, 11 und 12, welche sowohl bei der Bestimmung des Extraktes nach der direkten wie nach der Additionsmethode negative, z. T. sehr stark negative Werte für den totalen Extraktrest geben. Die Erklärung für dieses auffallende Verhalten ergibt sich unschwer aus ihrer chemischen Zusammensetzung, insbesondere ibrem hohen Gehalte an flüchtigen Säuren, welche sich im unveränderten natürlichen Citronensafte entweder gar nicht, oder, wie vorhin gezeigt, nur in Spuren vorfinden. Es ist bekannt, daß die Citronensäure unter der Einwirkung gewisser Spaltpilze zerstört und durch eine besondere Gärung in Essigsäure übergeführt wird, und zweifellos waren auch die vorliegenden Erzeugnisse, deren verdorbene Beschaffenheit sich schon durch den Geruch und Geschmack offenbarte, einer derartigen tiefgreifenden Zersetzung anheimgefallen. Eine solche folgt auch schon aus dem Umstande, daß ein absichtlicher Zusatz von Essigsäure, die ja von der Gesamtsäure in Abzug gebracht worden ist, das Bild der Analyse gar nicht stören würde, während im Verlaufe einer unkontrollierbaren Bakterientätigkeit Nebenprodukte entstehen können, die sich zurzeit dem chemischen Nachweise entziehen. Wir stehen nicht an, der uns privatim von Herrn Prof. Dr. Farnsteiner mitgeteilten Ansicht zuzustimmen, daß Aldehydbildung und dementsprechend zu hohe Ergebnisse bei der Zuckerbestimmung die Ursache sind, haben aber aus Rücksicht auf den genannten Forscher von einer Verfolgung der Frage Abstand genommen. Für den Zweck der vorliegenden Arbeit erscheint es ausreichend, darauf hinzuweisen, dab die Bestimmung der flüchtigen Säuren unter allen Umständen ausgeführt werden muß, da sonst schwere Irrtümer unvermeidlich sind. Auch für die Beurteilung der ersten 9, scheinbar ganz normalen Citronensäfte in Tabelle IV ist diese Tatsache von Bedeutung. Unter den 4 Proben, welche nennenswerte Mengen flüchtigex Säuren enthalten, nämlich No. 1, 2,6 und 8 waren No. 1, 6 und 8 uns ausdrücklich als mit 0,30 bezw. $0,15 \%$ Ameisensäure konserviert bezeichnet worden, während No. 2 frei von irgendwelchen Konservierungsmitteln sein sollte und tatsächlich Ameisensäure nicht enthielt. Bei dieser mußte also eine beginnende Essiggärung eingetreten sein und durch Vortäuschung eines zu hohen Zuckergehaltes das Bild der Analyse getrübt haben. Wir werden demnach diese Probe ausschalten und finden dann, daß die totalen Extraktreste der unverdorbenen Handelscitronensäfte aus 1905-er Citronen sich zwischen 1,255 und $0,539 \mathrm{~g}$ bewegen.

Selbstverständlich sind die niedrigen Werte Lührig's, der ja frische selbstgepreßte Säfte untersuchte, durch ähnliche Vorgänge nicht verursacht worden: Wohl aber liegt der Gedanke nahe, seine Analysen mit denjenigen der Sorrento-Citronen zu vergleichen. Das von ihm benutzte Ausgangsmaterial ist in verschiedenen Geschäften der Stadt Chemnitz angelsauft worden, ohne daß nähere Feststellungen über Sorte und Herkunft getroffen werden konnten. Bei den heutigen Verhältnissen des Großhandels, welcher die Städte mit ungeheuren gleichartigen Sendungen von Südfrüchten versorgt, und bei der außerordentlichen Úbereinstimmung der Lührig'schen Analysen (mit Ausnabme von Probe No.4) könnte man vielleicht annehmen, daß alle Früchte derselben Sorte angehören und zwar einer besonders saftreichen, welche wie die Sorrento-Citrone in der praktischen Fruchtpresserei nicht zur Verwendung kommt.

Ohne daher die Bedentung der Lührig'schen, wie unserer eigenen niedrigen 
Befunde für den totalen Extraktrest zu verkennen, möchten wir doch meinen, daß es nicht ratsam ist, die Anforderungen an Handelssäfte gar zu sehr herabzusetzen. Gewiß stimmen wir dem Ausspruche Lührig's bei, „daß es nicht ratsam ist, ohne ausgiebiges Material verallgemeinernde Grundsätze aufzustellen", glauben aber andererseits doch, daß nur der Anfänger, der noch nicht gelernt hat, die einzelnen Befunde im Rahmen der Gesamtanalyse zu verwerten, durch derartige Verallgemeinerung zu Irrtümern geführt werden kann.

Auffallend berührt es, daß derselbe Autor, der sich wenige Zeilen vorher als so entschiedener Gegner aller Grenzzahlen bekennt, in bezug auf Glycerin weniger skrupulös ist und eine unzweideutige Grenzzahl aufstellt, deren Gültigkeit keineswegs über jeden Zweifel erhaben ist. Nach unseren Analysen betrug der Gehalt an sog. Glycerin bei den selbstgepreßten Citronensäften, welche, nach dem geringen Zuckerrest zu urteilen, offenbar sehr stark vergoren waren, 0,057 bis $0,151 \mathrm{~g}$, und auch der Gehalt der Handelscitronensäfte überschritt diesen Wert nicht. Wenn demgegenüber Lührig in seinen Säften, deren hoher Zuckergebalt eine nennenswerte Gärung überhaupt ausgeschlossen erscheinen läßt, einmal bis zu $0,4 \%$ Glycerin fand, so erscheint diese eine Analyse nicht ausreichend, um darauf eine Grenzzahl zu begründen, sondern der Nachprüfung bedürftig.

Besonderes Interesse erregten uns die Angaben Lepère's, daß er bei der direkten Extraktbestimmung nahezu dieselben Werte wie bei der Additionsmethode erhalten habe, und obwohl seine Beobachtungen mit denjenigen Farnsteiner's, Lührig's und unseren eigenen nicht im Einklang standen, sind wir doch auch dieser Frage noch einmal näher getreten. Allerdings sind die starken Abweichungen zwischen den Befunden unserer Analysen vom Jahre 1904, durch welche Lepère zuerst zu einer Nachprüfung veranlabt worden war, durch die Umrechnung der erhaltenen Werte nach dem Additionsverfahren wesentlich verringert worden, nämlich um 0,254 bis $0,543^{1}$ ), aber. sie bleiben auch so noch mit den Resultaten Lepère's unvereinbar. Aus nachfolgender Zusammenstellung wird dies noch besser erhellen:

Tabelle V. Selbstgeprekte 1904-er Säte.

\begin{tabular}{|c|c|c|c|c|c|c|c|c|c|}
\hline \multirow[b]{2}{*}{ No. } & \multirow[b]{2}{*}{ Zucker } & \multicolumn{2}{|c|}{ Extrakt } & \multirow[b]{2}{*}{$\begin{array}{l}\text { Differenz } \\
z \text { wisehen } \\
a \text { und } b\end{array}$} & \multirow[b]{2}{*}{ No. } & \multirow[b]{2}{*}{ Zucker } & \multicolumn{2}{|c|}{ Extrakt } & \multirow[b]{2}{*}{$\begin{array}{l}\text { Differenz } \\
\text { zwisehen } \\
\text { a und }\end{array}$} \\
\hline & & $\begin{array}{c}a \\
a \mathrm{ar}=\mathrm{kt} \\
\mathrm{g}\end{array}$ & $\begin{array}{c}\text { b } \\
\text { nach dem } \\
\text { Additions- } \\
\text { verfabren } \\
\mathrm{g}\end{array}$ & & & & $\begin{array}{c}a \\
\text { direkt } \\
g\end{array}$ & $\begin{array}{c}\text { b } \\
\text { nach dem } \\
\text { Additions- } \\
\text { verfahren } \\
\mathrm{g} \\
\end{array}$ & \\
\hline 1 & 1,500 & 8,774 & 9,423 & $+0,649$ & 10 & 1,290 & 9.164 & 9,971 & $+0,807$ \\
\hline 2 & 1,490 & 8,782 & 9,459 & $+0,677$ & 11 & $0,54 \hat{0}$ & 9,139 & 9,667 & $+0,528$ \\
\hline 8 & 1,630 & 9,470 & 10,164 & $+0,694$ & 12 & 1,470 & 9,149 & 9,719 & $+0,570$ \\
\hline 4 & 1,490 & 10,007 & 10,652 & $+0,645$ & 13 & 0,040 & 8,128 & 8,361 & $+0,233$ \\
\hline 5 & 1,840 & 8,804 & 9,662 & $+0,858$ & 14 & 1,820 & 10,014 & 10,740 & $+0,726$ \\
\hline 6 & 0,060 & 7,635 & 7,839 & $+0,204$ & 15 & 0,011 & 8,228 & 8,294 & $+0,066$ \\
\hline 7 & 1,340 & 9,415 & 9,726 & $+0,311$ & 16 & 2,550 & 10,164 & 10,886 & $+0,722$ \\
\hline 8 & 1,680 & 9,199 & 9,767 & $+0,568$ & 17 & 0,080 & 8,452 & 8,607 & $+0,155$ \\
\hline 9 & 0,050 & 8,167 & 8,316 & $+0,149$ & & & & & \\
\hline
\end{tabular}

1) Die uns theoretisch unmöglich erscheinende Tatsache, daß L ührig zwischen den nach der ursprünglichen Methode $\mathbf{F}$ arnsteiner's und den nach dem Additionsverfahren berechneten Extraktresten z. T. Differenzen von nahezu Null angibt, findet ihre Erklärung durch kleine Rechenfehler in der letzten Spalte seiner Tabelle I, die übrigens für das Gesamtresultat der Arbeit ohne Bedeutung sind. 


\begin{tabular}{|c|c|c|c|c|c|c|c|c|c|}
\hline \multirow[b]{2}{*}{ No. } & \multirow[b]{2}{*}{ Zucker } & \multicolumn{2}{|c|}{ Extrakt } & \multirow[b]{2}{*}{$\begin{array}{c}\text { Differenz } \\
\text { zwischen } \\
\text { a und b }\end{array}$} & \multirow[b]{2}{*}{ No. } & \multirow[b]{2}{*}{$\begin{array}{c}\text { Zucker } \\
\mathrm{g} \\
\end{array}$} & \multicolumn{2}{|c|}{ Extrakt } & \multirow[b]{2}{*}{$\begin{array}{l}\text { Differenz } \\
z \text { wisehen } \\
a \text { und } b\end{array}$} \\
\hline & & $\begin{array}{c}\underset{a}{d i r e k t} \\
g\end{array}$ & $\begin{array}{c}\mathrm{b} \\
\text { nach dem } \\
\text { Additions- } \\
\text { verfahren } \\
\mathrm{g}\end{array}$ & & & & $\begin{array}{c}\mathbf{a} \\
\text { direkt } \\
\mathrm{g}\end{array}$ & $\begin{array}{l}\text { b } \\
\text { naeh dem } \\
\text { Additions- } \\
\text { verfahren } \\
\mathbf{g}\end{array}$ & \\
\hline \multicolumn{5}{|c|}{ Selbstgepreß te 1905-er Säfto. } & \multicolumn{5}{|c|}{ 1905-er Handels-Citronensäfte. } \\
\hline 1 & 0,190 & 6,619 & 6,448 & $-0,161$ & 1 & 1,354 & 8,040 & 8,282 & $+0,242$ \\
\hline 2 & 0,220 & 8,348 & 8,445 & $+0,097$ & 2 & 0,160 & 8,313 & 8,100 & $-0,213$ \\
\hline 3 & 0,110 & 7,104 & 7,285 & $+0,169$ & 3 & 0,123 & 7,004 & 7,077 & $-0,073$ \\
\hline 4 & 0,240 & 8,088 & 7,899 & $-0,189$ & 4 & 0,488 & 8,411 & 8,473 & $+0,062$ \\
\hline 5 & 0,110 & 8,050 & 7,770 & $-0,280$ & 5 & 1,006 & 7,337 & 7,598 & $+0,261$ \\
\hline 6 & 0,034 & 6,011 & 5,931 & $-0,080$ & 6 & 0,642 & 8,497 & 8,390 & $-0,107$ \\
\hline 7 & 0,033 & 5,994 & 5,803 & $-0,191$ & 7 & 0,128 & $7,6 \geq 3$ & 7,697 & $+0,074$ \\
\hline 8 & 0,097 & 7,011 & 6,923 & $-0,088$ & 8 & 1,310 & 8,600 & 8,242 & $-0,358$ \\
\hline 9 & 0,033 & 6,183 & 6,214 & $+0,081$ & & & & & \\
\hline
\end{tabular}

Nach diesen Zahlen sind die Differenzen zwischen den beiden Extraktwerter doch recht bedeutend. Zwar fällt an den Analysen des Vorjahres auf, daß die Unterschiede bei den völlig vergorenen Säften am kleinsten sind und in einzelnen Fällen nahezu Null betragen, und auch die wesentlich geringeren Differenzen in der zweiten Tabellenspalte scheinen die naheliegende Annahme, daß der Zuckergehalt die Ursache der Abweichungen sei, zu bestätigen. Aber dafür finden wir hier wieder, wie auch Lührig, eine ganze Anzahl negativer Werte, und eine allgemeine Regel läßt sich daher nicht wohl aufstellen. Man wird also einstweilen wohl oder übel von der Verwertung des direkt bestimmten Extraktes absehen müssen.

Zum Schluß noch einige Worte über die von Küttner und Ullrich empfohlene Ammoniakprobe. Wie wir bereits im Vorjahre mitteilten, nahmen die von uns selbst gepreßten Säfte, mit Ausuahme der aus Verdelli-Citronen hergestellten, auf Zusatz von Ammoniak keine braune, sondern nur eine etwas dunklere gelbe Farbe an, und auch unser diesjähriges Analysenmaterial verhielt sich in ganz analoger Weise. Eine Erklärung für das abweichende Verhalten echter Handelscitronensäfte vermochten wir nicht zu geben, nachdem sich herausgestellt hatte, dab die Eiweiß- und Pektinstoffe an der Reaktion jedenfalls unbeteiligt sind. Um so plausibler erschien uns die von Frl. Dr. Köpcke ${ }^{1}$ ) geäußerte Ansicht, daß der höhere Eisengehalt der durch Kieselgur oder Speckstein filtrierten Handelssäfte die Ursache der Erscheinung sei, und wir haben daher zur Nachprüfung dieser Auffassung den Eisengehalt einiger selbst hergestellter Citronensäfte sowie eines Handelsproduktes, welches die Reaktion in besonders starker Weise lieferte, bestimmt.

Die Analyse ergab folgende Werte:

$100 \mathrm{ccm}$ Saft enthielten Eisen (Fe):

$\begin{array}{ccccc}\text { I. } & \text { VI. } & \text { VII. } & \text { VIII. } & \text { Handelssaft } \\ 0,91 \mathrm{mg} & 2,21 \mathrm{mg} & 2,12 \mathrm{mg} & 3,33 \mathrm{mg} & 5,48 \mathrm{mg}\end{array}$

Spricht schon der verhältnismäBig geringe Mehrgebalt an Eisen in dem Handelscitronensaft nicht gerade dafür, daf in ihm die Ursache der Braunfärbung liegt, sa.

1) Pharm. Centralhalle 1905, 47, 974. 
wird diese Annahme noch unwahrscheinlicher gemacht durch die Beobachtung, daß der Saft No. VI, welcher anfangs auf Zusatz von Ammoniak nur gelb wurde, nach mehrwöchentlicher Aufbewahrung trotz seines geringen Eisengehaltes die Reaktion in schönster Weise zeigte. Außerdem fanden wir zwar in Übereinstinmung mit Köpcke, daß ein Zusatz von Eisenchlorid die Braunfärbung hervorruft, nicht aber ein solcher von Eisencitrat. Wir möchten daher unsere Meinung dahin äußern, daß andere Bestandteile organischer Natur, etwa die auch von Köpcke erwähnten Gerbstoffe, oder harzartige, im Laufe der Zeit entstehende, Verbindungen als Träger der Reaktion anzusprechen sind. Daf diese Verdelli-Citronen die Erscheinung besonders deutlich zeigen, könnte an ihrer abweichenden Zusammensetzung, die sich schon in ihrer mehr grünlichen Färbung äußert, liegen. Jedenfalls kann der Chemismus der Reaktion auch jetzt noch nicht als völlig aufgeklärt angesehen werden und Vorsicht bleibt ihr gegenüber geboten.

Im Hinblick auf die vielen, bezüglich der Citronensaftanalyse noch immer zu lösenden Fragen können wir uns nur dem Wunsche Lührig's anschließen, daß sich recht zahlreiche Fachgenossen an dieser mühsamen, die Kraft des einzelnen übersteigenden Arbeit beteiligen, und möchten es als eine dankenswerte Aufgabe der Kommission für Fruchtsäfte bezeichnen, nach dem Muster der Himbeersaft-Statistik für 1905 möglichst umfangreiches Material zu sammeln.

Dresden, im Mai 1906.

\title{
Zur Kenntnis der Frucht von Capsicum annuum L.
}

\author{
Von
}

\section{A. Nestler.}

Mitteilung aus derk. k. Untersuchungsanstalt für Lebensmittel in Prag (Deutsche Universität).

Mit 1 Tafel.

\section{Krystalle der Sekretdrüsen.}

Die Entstehung der wirksamen Substanz der Paprikafrucht, des Capsaïcins findet nach den Untersuchungen von A. Meyer ${ }^{1}$ ) und $\mathrm{H}$. Molisch ${ }^{2}$ ) - darüber kann trotz anderer geäußerten Ansichten kein Zweifel bestehen - einzig und allein in den Fruchtscheidewänden und zwar in den hier mehr oder weniger zahlreich auftretenden Hautdrüsen statt. Wenn man eine reife Frucht von Capsicum annuum, die man vor starken Erschütterungen bewahrt hat, vorsichtig aufschneidet, um ein Herumrollen losgelöster Samen und damit eine Übertragung der capsaïcinhaltigen Substanz auf die Innenepidermis der Fruchthaut zu verhüten, und darauf die Fruchthaut durch den Geschmack prüft, so findet man, daß sie infolge des reichen Gehaltes an reduzierendem Zucker süß schmeckt und keine Spur einer brennenden Wirkung zeigt. Da wir bisher kein an der reinen Substanz erprobtes, mikroskopisches Reagens

1) A. Meyer, Der Sitz der scharf schmeckenden Substanz im Spanischen Pfeffer. Pharm. Ztg. 1889, 16, 130.

2) H. Molis ch, Grundrifs einer Histochemie der pflanzlichen Genußßmittel. 1891, 53. 